\title{
Are GPs under-investigating older patients presenting with symptoms of ovarian cancer? Observational study using General Practice Research Database
}

\author{
AR Tate ${ }^{*, 1}$, A Nicholson' and JA Cassell' \\ 'Division of Public Health and Primary Care, Brighton and Sussex Medical School, Falmer, Brighton BNI 9PH, UK
}

\begin{abstract}
BACKGROUND: Recent studies suggest that older patients in the United Kingdom are not benefiting as much from improvements in cancer treatments as their younger counterparts. We investigate whether this might be partly due to differential referral rates using ovarian cancer as an example.

METHODS: From the General Practice Research Database (GPRD), we identified all women aged 40-80 years on I June 2002 with a Read code for ovarian cancer between I June 2002 and 3I May 2007. Using these records, we compared the GPRD incidence of ovarian cancer with rates compiled from the UK cancer registries and investigated the relationship between age and coded investigations for suspected ovarian cancer.

RESULTS: The GPRD rates peaked earlier, at 70-74, and were lower than registry rates for nearly all ages particularly for patients over 59. The proportion investigated or referred by the GP decreased significantly with age and delays between first coded symptom and investigation showed a U-shaped distribution by age.

CONCLUSIONS: GPS appear to be less likely to recognise and to refer patients presenting with ovarian cancer as they get older. If our findings extend to other cancers, lack of or delays in referral to secondary care may partly explain poor UK cancer mortality rates of older people.
\end{abstract}

British Journal of Cancer (2010) I 02, 947-95I. doi:I0.1038/sj.bjc.6605593 www.bjcancer.com

Published online 2 March 2010

(c) 2010 Cancer Research UK

Keywords: primary care delay; secondary care referral; patient age

Cancer is a disease that predominantly affects older people and in our ageing population increasing numbers of people are going to be affected by this disease. However, significant advances in cancer treatment seem not to be benefiting older people as much as they could. A recent study by the EUROCARE group found that the gap in survival between older and younger European cancer patients is getting wider (Quaglia et al, 2009). The United Kingdom, which already has lower cancer survival rates than most of Europe (Berrino et al, 2007), seems to be doing particularly badly where older people are concerned. A comparison of data from the World Health Organization registry database suggests little or no improvement in cancer mortality rates for the UK elderly during the last decade, and that cancer mortality rates for patients over 64 compare badly with other Northern and Western European countries and the United States (Moran and Moeller, 2009). The cause of this discrepancy is unclear, but as most patients experiencing cancer-related symptoms in the United Kingdom present first to primary care (Kirwan et al, 2002; Allgar and Neal, 2005), it is possible that older cancer patients are being managed differently from their younger counterparts by their GP.

*Correspondence: Dr AR Tate; E-mail: r.tate@bsms.ac.uk Received 19 November 2009; revised 2 February 2010; accepted 3 February 20I0; published online 2 March 2010
The existence of large primary care databases of electronic patient records provides the opportunity to investigate GPs management of cancer patients in the United Kingdom using a large cohort of patients. We have already been using the General Practice Research Database (GPRD) to investigate primary care delay using ovarian cancer as an example (Tate et al, 2009), and have shown that the time between the first recorded relevant symptom and first recorded referral is typically around 10 weeks.

The purpose of this study is to investigate whether patient age is a factor in this delay and in particular to investigate the effect of patient age on:

1. The incidence of coded diagnoses for ovarian cancer in general practice (compared with the UK cancer registry rates).

2. Investigation or referral before diagnosis of ovarian cancer.

3. The delay between a patient first reporting an ovarian cancerrelated symptom and first recorded referral or investigation.

\section{MATERIALS AND METHODS}

\section{Data and measures used}

Subjects The GPRD (GPRD, 2009) is one of the largest primary care databases in the United Kingdom. It contains anonymised longitudinal data on a representative sample of the UK population. Records are being collected on over 3.6 million currently registered 
patients (approximately 13 million total) who are registered for care in general practice from around 488 primary care practices throughout the United Kingdom. These records are created during consultations or when processing correspondence, and are widely used in research on disease epidemiology, drug safety and adverse drug reactions (Hansell et al, 2004; Majeed et al, 2008; Jones et al, 2009).

This study forms part of an ongoing investigation of prognostic symptoms and primary care delays in ovarian cancer (Tate et al, 2009). The data set was provided under the MRC licence scheme and access was approved by the Independent Scientific Advisory Committee (Protocol 07_069). The target population consisted of all females between 40 and 80 years of age (inclusive) who were alive and registered with a GPRD contributing practice on 1 June 2002. From this population, all women with an incident diagnosis of ovarian cancer recorded during 1 June 2002-31 May 2007 were identified $(N=1166)$. Women with a previous diagnosis of ovarian cancer were excluded. An incident diagnosis of ovarian cancer was defined by a first Read code recorded in the patient's clinical or referral record, that is, B440.00 (Malignant neoplasm of ovary), B440.11 (Cancer of ovary) or B44.00 (Malignant neoplasm of ovary and other uterine adnexa).

The UK cancer registry incidence rates for 2002-06 were provided by the Statistical Information Team at Cancer Research UK (CRUK, 2009). These rates represent the most recent statistics that are currently available. To compare these rates with those recorded in the GPRD, we used only those (1057) patients who were coded as being diagnosed with ovarian cancer between 1 June 2002 and 31 December 2006. Denominator data for the female GPRD patient population were used to calculate rates of recorded ovarian cancer. These included information on the number of patients registered in the GPRD and equivalent person-years stratified by calendar year, age and practice.

For the description of investigations/referrals, we selected all ovarian cancer cases (from the 1166 cases recorded during 1 June 2002-31 May 2007) that had been registered for at least 1 year before the GP had recorded a diagnosis $(n=1107)$. For the investigation of delays, we used only those patients who had been recorded as having a relevant investigation and who had been registered for at least 1 year before the date of first recorded investigation.

\section{MEASURES}

Read codes for a relevant investigation or referral for ovarian cancer were identified and grouped into the following categories: (1) oophorectomy, (2) laparoscopy, (3) laparotomy, (4) referral to gynaecologist, (5) CA125 test, (6) CAT scan and (7) ultrasound. Of these, 1-3 were subcategorised as invasive investigations and 4-7 as non-invasive investigations. Although 1-3 are often used to treat ovarian cancer, they also often serve as an investigation and thus may be the first recorded procedure. Read codes for the most commonly recorded ovarian cancer-related symptoms were identified and grouped into the following 11 categories: (1) abdominal pain, (2) pelvic pain, (3) back pain, (4) abdominal distension/bloating, (5) indigestion, (6) nausea and vomiting, (7) constipation/change in bowel habit, (8) urogenital symptoms, (9) appetite weight, (10) tiredness and (11) breathing problems. Further details of how these measures were obtained, together with the code list for symptoms, are published elsewhere (Tate et al, 2009).

\section{Data analysis}

Rates of incident-coded diagnosis were calculated by dividing the number of first-time diagnosis codes for ovarian cancer by the number of person-years of women registered in the GPRD during the corresponding time period and stratified by 5-year age bands.

The percentage of each age group having a relevant referral or investigation for ovarian cancer was calculated for each type of investigation by dividing the number of cases with at least one code for that investigation in the year before and including the diagnosis date, by the total number of cases in that age group.

The delay between a symptom being first recorded and investigation was calculated by estimating the time from the GP first recording the symptom to the first relevant investigation.

The binomial test was used to test for differences between the proportions reported in the GPRD and the cancer registries and a non-parametric trend test (Stata's nptrend) was used to test for trends by age (in years). Data management was undertaken using MySql (http:/www.mysql.com) and statistical analyses were performed using Stata 10 (Stata Corporation, College Station, TX, USA).

\section{RESULTS}

\section{Comparison with cancer registry data}

The rate of recorded diagnosis of ovarian cancer in the GPRD was significantly lower than the UK incidence rate for nearly all age groups, with the difference being greatest in those aged $>60$ years (Table 1). The GPRD rates peaked at $70-74$, in contrast to the registry-based incidence rate in which the highest rate was in the $80-84$ age group.

\section{Investigations}

Of the 1107 cases that were registered with a GPRD practice for at least 1 year before the date of first coded diagnosis, 810 (73\%) were

Table I The rates of diagnosis codes in the GPRD between June 2002 and December 2006 compared with the UK registry-based rate for $2002-06$ (CRUK, 2009)

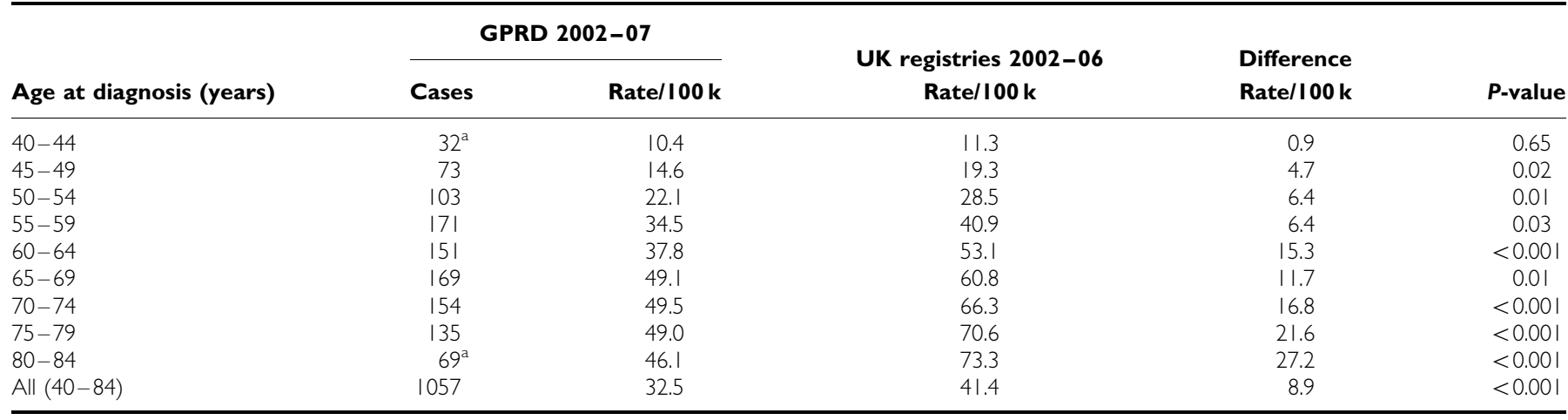

Abbreviation: GPRD, General Practice Research Database. ${ }^{a}$ Owing to the sampling strategy of selecting patients aged between 40 and 80 on I June 2002 , there are fewer cases than would normally be expected in the youngest and oldest age bands. 
Table 2 Proportion of cases having relevant surgery or investigation for ovarian cancer in the year before diagnosis by age

\begin{tabular}{|c|c|c|c|c|c|c|c|c|c|c|}
\hline Age at diagnosis (years) & $40-44$ & $45-49$ & $50-54$ & $55-59$ & $60-64$ & $65-69$ & $70-74$ & $75-79$ & $80-84$ & Total \\
\hline Number of cases & 31 & 69 & 105 & 177 & 164 & 174 & 171 & 139 & 77 & 1107 \\
\hline \multicolumn{11}{|c|}{ Proportion having invasive investigation } \\
\hline Oophorectomy* & 0.52 & 0.33 & 0.3 & 0.29 & 0.25 & 0.2 & 0.18 & 0.12 & 0.16 & 0.23 \\
\hline Laparotomy & 0.06 & 0.13 & 0.1 & 0.12 & 0.1 & 0.09 & 0.13 & 0.14 & 0.1 & 0.11 \\
\hline Laparoscopy & 0.03 & 0.04 & 0.03 & 0.03 & 0.02 & 0.02 & 0.02 & 0.03 & 0.04 & 0.03 \\
\hline Any of above* & 0.55 & 0.42 & 0.36 & 0.38 & 0.3 & 0.26 & 0.28 & 0.22 & 0.26 & 0.31 \\
\hline \multicolumn{11}{|c|}{ Proportion referred to gynaecologist or having non-invasive investigation } \\
\hline Gynaecology* & 0.48 & 0.57 & 0.45 & 0.47 & 0.43 & 0.38 & 0.32 & 0.36 & 0.35 & 0.41 \\
\hline CAI 25 & 0.19 & 0.29 & 0.32 & 0.19 & 0.24 & 0.27 & 0.24 & 0.22 & 0.17 & 0.24 \\
\hline CAT scan & 0.06 & 0.13 & 0.2 & 0.15 & 0.14 & 0.18 & 0.15 & 0.17 & 0.17 & 0.16 \\
\hline Ultrasound* & 0.45 & 0.39 & 0.39 & 0.35 & 0.29 & 0.28 & 0.29 & 0.24 & 0.27 & 0.31 \\
\hline Any of the above $4 *$ & 0.68 & 0.78 & 0.75 & 0.67 & 0.66 & 0.68 & 0.61 & 0.6 & 0.57 & 0.66 \\
\hline Any of the above* & 0.9 & 0.81 & 0.8 & 0.75 & 0.76 & 0.75 & 0.68 & 0.65 & 0.62 & 0.73 \\
\hline
\end{tabular}

Abbreviation: CAT, computed tomography. *With $P$-value of $<0.05$ for negative trend with age (all items with an asterisk represent a $P$-value of $\leqslant 0.01$ ).

coded as having at least one relevant investigation or referral to a gynaecologist in the year before diagnosis. This proportion decreased with age for most types of investigation (Table 2), with $82 \%$ aged $<55$ years having had at least one recorded investigation compared with $75 \%$ aged between 55 and 69 years and $66 \%$ of those aged $\geqslant 70$ years. The decreases with age were significant for both invasive and non-invasive investigations $(P \leqslant 0.001)$.

\section{Delay between reporting a symptom and investigation}

The four most commonly recorded ovarian cancer-related symptom groups in the year before coded diagnosis or first investigation (if recorded) were abdominal pain (43\%), urinary/ genital problems (23\%), abdominal distension (22\%) and constipation (21\%), with the next most common being nausea/ vomiting (12\%). Of the 810 patients recorded as having at least one investigation, 804 had been registered a full year before the first recorded investigation date. Of the latter, 640 (77\%) were coded with at least one relevant symptom during the year before investigation and $545(68 \%)$ with one of the four most common symptoms. Of those (297 cases) with no recorded investigation, $72 \%$ had at least one relevant symptom and $64 \%$ had one of the four most common symptoms in the year before coded diagnosis. There was a slight increase in symptoms with increased age, but the trend was not significant at the 0.05 level $(P=0.08$ for any symptom and $P=0.38$ for any of the most common four).

The median (IQR) time in weeks between the first recorded relevant symptom and first investigation or referral to the gynaecologist was $10.5(2,32)$ for the 11 relevant symptoms and $7(1,23)$ for the four most common symptoms. Figure 1 shows the distribution of time between first reporting any one of the relevant symptoms and referral for each age category. The median delays have a U-shaped distribution, with the younger and older patients having longer delay than those in the middle age groups. Although the distribution of delay in the younger age group was similar to that of older cases, the overall trend was for delays to increase with age $(P=0.003)$. The biggest differences were between those aged $\geqslant 70$ years compared with those aged between 45 and 69 years. A similar pattern was observed for the four most common symptoms (data not shown).

\section{DISCUSSION}

This study not only shows that age has a major effect on how GPs manage women with ovarian cancer, but also shows that

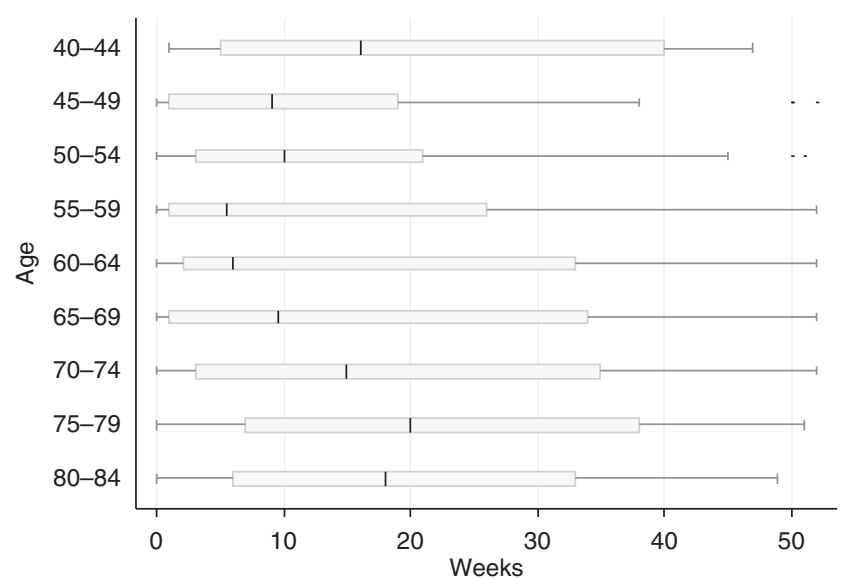

Figure I The distribution of time in weeks before a patient first reporting any ovarian cancer symptom and being referred for a relevant investigation, by age.

they are less likely both to code a diagnosis and to refer patients for gynaecological investigation when they are older. Our results also suggest that when GPs do refer elderly patients for investigation, they are slower to do so than for their middle-aged counterparts.

This study uses recently recorded information from a large cohort of patients representative of the UK population. Large primary care databases such as the GPRD enable the study of patterns of management of patients in primary care in a large unselected cohort of patients and are ideal for studying relatively rare diseases such as ovarian cancer. However, a disadvantage of using records where data are collected for clinical rather than research purposes is that coding may be incomplete. This was shown in our previous study (Tate et al, 2009), in which we found inaccuracies in the dating of diagnosis, and again in this study in which we find that rates of coded diagnosis of ovarian cancer are 9\% lower than the UK incidence rates.

This study was based on coded data and did not use information from the free text fields, which, owing to the costs of anonymisation, was not available for this study. Thus, we may have missed diagnoses, referrals and symptoms that GPs chose to record only in the free text. Although there is no reason to suppose that patient age affects how GPs use the free text fields, this is an important 
area that warrants further investigation. However, the context of consultations (e.g., home visit, telephone consultation) may have an age-related influence on recording practices and this could be a source of confounding in our analysis, as could investigations for other conditions. Read codes often do not provide information on the type of investigation or the site of the body where it is carried out. So, for example, a code for imaging may relate to an investigation for suspected ovarian cancer, or for some other complaint. As a result, and also because older people are more likely to have other complaints, we considered only investigations that we were almost sure would pertain to an investigation for suspected ovarian cancer.

The sampling strategy of setting the patient's age at the start of the study meant that there were fewer cases in the lower and upper age groups than would be expected if we had sampled over the full age range for the whole study period; thus, for example, there were no 40 year olds after May 2003 and no 84 year olds until June 2006, and thus the $40-44$ and $80-84$ age bands are overrepresented by older and younger patients, respectively. We included these age bands in our results for completeness. The start date of June 2002 meant that in the comparison with the cancer registry data, which covered the full year, the first part of 2002 had no GPRD data. However, we believe that this will have little impact on the results as incidence rates are not changing rapidly.

As we only looked at one type of cancer, we do not know if our results will generalise to other cancers. However, ovarian cancer would seem to be a good example to study as it is one of the most common cancers experienced by older women, and its prognosis is greatly improved if it is diagnosed at an early stage (Falandry et al, 2008). It was one of the cancers found to have worse prognosis in the United Kingdom than in other European countries (Berrino et al, 2007).

To our knowledge, this is the first study to investigate agerelated differences between the incidence of codes in general practice records for ovarian cancer and the cancer registries. However, other studies have reported similar overall discrepancies between GP recording and registry data, for all cancers (Pascoe et al, 2008), and for ovarian cancer patients (Hamilton, 2009). It is interesting that both these studies included information from the free text. Haynes et al (2009) found that rates for solid cancers coded in the Health Improvement Network database were lower than those in the cancer registry and hypothesised that this could be because their study included only cases with an unambiguous Read code for ovarian cancer, whereas GPs may prefer to use a more ambiguous code. This might also be true for our study and could explain the lower overall rate.

The increasing disparity in incidence with age may be more difficult to explain. One possible reason may be that GPs are less motivated to record cancer diagnoses in older people if they have other serious illnesses. Alternatively, recording details of the disease could be deemed to be less important for older people - it has been found that the stage or grade of tumour in cancer registry data is more likely to be missing if the patient is elderly (De Rijke et al, 1998; Adams et al, 2004). Another possible reason, which might partly explain the earlier peak in recorded ovarian cancer in the GPRD, could be a possible time lag between recording a diagnosis in GP records and informing the cancer registries (data for which come mostly from other sources including hospitals and the death registry). There may also be non-clinical reasons for differential patterns of recording; for example, older patients may be more likely to consult at home or be diagnosed or referred to a hospital visit for some other purpose.

Our results on investigations and referral agree with other reports that have found that patients are less likely to be investigated or treated for cancer if they are elderly (e.g., Turner et al, 1999; Peake et al, 2003; Bouchardy et al, 2007). However, most other studies are hospital based and do not investigate what happens in primary care.
Few studies have investigated the relationship between delays in cancer referrals and patient age and those that have show conflicting results (Neal and Allgar, 2005). To our knowledge, the only other study to look at ovarian cancer was that of Neal and Allgar (2005), which was based on a large NHS survey of data collected from patients with one of six types of cancer discharged from hospital between July 1999 and June 2000. They found, in contrast to our results, that referral delay (for ovarian and a number of other cancers) decreased with patient age. However, they used a different methodology from our study; the data were based on patient reports that are prone to recall and selection bias, which may be greater in older people. For example, older people tend to be more forgetful and those who had experienced long delays may have died before they could be included in the survey. Other differences from our study were that the time period between first reporting a symptom and delay was unspecified and that mean rather than median delays were compared. Another UK-based study, which examined factors influencing delay in colorectal cancer (Robertson et al, 2004), found a similar age-related distribution to ours, with the fastest referrals being in the 50-64 age group. This same study also examined breast cancer delays and found that these decreased with increasing age. The authors suggest that differences in delay for the different cancers depend on how the tumour presents and also how common (and thus predictive) the symptoms are in the group that are presenting. As with ovarian cancer, colorectal cancer has nonspecific symptoms that may include abdominal distension and abdominal pain. This may explain why their findings are similar to ours at least for the female cases, as these symptoms are commonly experienced by women without cancer before and during the menopause and then again in old age.

The lower rates of recording compared with cancer registries suggest that primary care data may be less fit for the purpose of measuring incidence in older age groups. It is important that the quality of records is high in the elderly if we are to maximise health gain from electronic patent records through disease surveillance and monitoring and improvement of care. Despite this limitation, this study, based on recent information from GP surgeries, suggests that there is a decline in recorded investigation and referral in older women for ovarian cancer. Such delays could be an important cause of avoidable morbidity and mortality, and if our results are generalisable to other cancers, they could contribute to the lower survival rates and higher mortality rates experienced in the United Kingdom compared with other European countries.

The discrepancies we have shown in the apparent incidence of ovarian cancer in the GPRD, as compared with the cancer registries, have implications for further records-based research on care pathways and on epidemiological research in this field. We are planning future work on the different strategies for investigation used by GPs for different age groups. Further work is also needed to explore whether these findings generalise to other cancers and the extent of possible confounding by differential recording practices in primary care for the elderly.

\section{ACKNOWLEDGEMENTS}

We thank the Statistical Information Team, Cancer Research UK, 2009 , for providing the cancer registry statistics. This work was supported by the Wellcome Trust $(086105 / \mathrm{Z} / 08 / \mathrm{Z})$. This study is based, in part, on data from the Full Feature General Practice Research Database obtained under licence from the UK Medicines and Healthcare Products Regulatory Agency. However, the interpretation and conclusions contained in this study are those of the authors alone. Access to the GPRD database was funded through the Medical Research Council's licence agreement with MHRA. 


\section{REFERENCES}

Adams J, Audisio R, White M, Forman D (2004) Age-related variations in progression of cancer at diagnosis and completeness of cancer registry data. Surg Oncol 13(4): 175-179

Allgar VL, Neal RD (2005) General practitioners' management of cancer in England: secondary analysis of data from the National Survey of NHS Patients - cancer. Eur J Cancer Care 14(5): 409-416

Berrino F, Angelis RD, Sant M, Rosso S, Lasota MB, Coebergh JW, Santaquilani M (2007) Survival for eight major cancers and all cancers combined for European adults diagnosed in 1995-99: results of the EUROCARE-4 study. Lancet Oncol 8(9): 773-783

Bouchardy C, Rapiti E, Blagojevic S, Vlastos A-T, Vlastos G (2007) Older female cancer patients: importance, causes, and consequences of undertreatment. J Clin Oncol 25(14): $1858-1869$

CRUK (2009) (accessed on 30 September 2009) Cancer Research UK: Cancer Stats. http://info.cancerresearchuk.org/cancerstats/

De Rijke J, Schouten L, Volovics A, Van der Putten H (1998) Age-specific differences in treatment and survival of ovarian cancer patients in the province of Limburg, the Netherlands, 1986-92. Int J Gynecol Cancer 8(2): $150-157$

Falandry C, You B, Morel-Soldner I, Bonnefoy M, Pujade-Lauraine E, Freyer G (2008) Ovarian cancer in the elderly. Oncologie 10(1): 3-7. 8th Biennale Monegasque Cancer Meeting, Monte Carlo, Monaco, 2008

GPRD (2009) GPRD. Excellence in public health research. http://www.gprd.com

Hamilton W (2009) The caper studies: five case-control studies aimed at identifying and quantifying the risk of cancer in symptomatic primary care patients. $\mathrm{Br} J$ Cancer 101(S2): S80-S86

Hansell AL, Lam KA, Richardson S, Visick G, Soriano JB (2004) Medical event profiling of COPD patients. Pharmacoepidemiol Drug Saf 13(8): 547 - 555

Haynes K, Forde KA, Schinnar R, Wong P, Strom BL, Lewis JD (2009) Cancer incidence in the health improvement network. Pharmacoepidemiol Drug Saf 18(8): $730-736$

Jones R, Charlton J, Latinovic R, Gulliford MC (2009) Alarm symptoms and identification of non-cancer diagnoses in primary care: cohort study. Br Med J 339: 491 - 493

Kirwan JMJ, Tincello DG, Herod JJO, Frost O, Kingston RE (2002) Effect of delays in primary care referral on survival of women with epithelial ovarian cancer: retrospective audit. $\mathrm{Br}$ Med J 324(7330): 148-151
Majeed A, Car J, Sheikh A (2008) Accuracy and completeness of electronic patient records in primary care. Fam Pract 25(4): 213-214

Moran A, Moeller H (2009) Poor progress in cancer control in elderly in UK. In: Proceedings of the 2009 NCIN Conference. National cancer Intelligence Network. pp 1-2. http://www.ncin.org.uk/about/conference/ 2009/11_oral_AMoran.shtml

Neal R, Allgar V (2005) Sociodemographic factors and delays in the diagnosis of six cancers: analysis of data from the 'National Survey of NHS Patients: Cancer'. Br J Cancer 92(11): 1971 - 1975

Pascoe SW, Neal RD, Heywood PL, Allgar VL, Miles JN, Stefoski-Mikeljevic J (2008) Identifying patients with a cancer diagnosis using general practice medical records and Cancer Registry data. Fam Pract 25(4): $215-220$

Peake M, Thompson S, Lowe D, Pearson M (2003) Ageism in the management of lung cancer. Age Ageing 32(2): 171-177

Quaglia A, Tavilla A, Shack L, Brenner H, Janssen-Heijnen $M$, Allemani C, Colonna M, Grande E, Grosclaude P, Vercelli M, Eurocare Working Group (2009) The cancer survival gap between elderly and middle-aged patients in Europe is widening. Eur J Cancer 45(6, Sp. Iss. SI): $1006-1016$

Robertson R, Campbell N, Smith S, Donnan P, Sullivan F, Duffy R, Ritchie L, Millar D, Cassidy J, Munro A (2004) Factors influencing time from presentation to treatment of colorectal and breast cancer in urban and rural areas. Br J Cancer 90(8): 1479-1485

Tate AR, Martin AGR, Murray-Thomas T, Anderson SR, Cassell JA (2009) Determining the date of diagnosis - is it a simple matter? The impact of different approaches to dating diagnosis on estimates of delayed care for ovarian cancer in UK primary care. BMC Med Res Methodol 9

Turner N, Haward R, Mulley G, Selby P (1999) Cancer in old age - is it inadequately investigated and treated? $\mathrm{Br}$ Med J 319(7205): 309-312

(c) (1) (2) This work is licensed under the Creative Commons BY NC SA Attribution-NonCommercial-Share Alike 3.0 License. To view a copy of this license, visit http://creativecommons.org/ licenses/by-nc-sa/3.0/ 